\title{
Petit essai pour montrer que la polysémie n'est pas un sens interdit
}

\author{
Georges Kleiber \\ Université Marc Bloch de Strasbourg \& EA 1339 LILPA-Scolia \\ kleiber@umb.u-strasbg.fr \\ Il n'y a qu'un sens, mais il est ... multiple
}

\section{Introduction}

Il n'est guère besoin de le souligner : les affaires de la polysémie sont aujourd'hui, à l'image même du concept, ambiguës ${ }^{1}$. Un seul point semble faire l'unanimité : tout le monde (ou presque) accepte qu'une expression linguistique puisse présenter une variation d'interprétations selon le contexte. Mais faut-il y voir de la polysémie ou non ? C'est là que les analyses et les réponses s'avèrent discordantes et qu'il convient de faire le point. Non pas en proposant une nième théorie, forcément "copernicienne », de la polysémie, mais en (ré)examinant, dans le droit fil de nos travaux antérieurs (Kleiber, 1996, 1999, 2000, 2005 et 2006), les tenants et les aboutissants de l'affaire.

Nous nous proposons de reprendre le problème de la multiplicité de sens associée à une unité lexicale pour essayer de voir quelles sont les propriétés que doivent présenter de tels sens pour mériter le label de polysémie ou non. Nous le ferons en deux étapes : après un tour de chauffe observationnel général, nous mettrons en avant dans la première partie les deux conditions auxquelles doivent satisfaire les différentes lectures d'une unité lexicale pour pouvoir être considérées comme étant des sens de l'unité lexicale ellemême : la non-unifiabilité et l'autonomie par rapport au discours. L'hypothèse que nous défendrons est que la variation interprétative d'une unité lexicale ne peut être polysémique ${ }^{2}$, c'est-à-dire portée au crédit de l'unité lexicale, que si les différentes lectures en jeu ont deux propriétés particulières : elles doivent être, d'une part non unifiables, et, d'autre part, robustes ou fortes, c'est-à-dire autonomes par rapport aux discours dans lesquels elles se manifestent. Nous essaierons, comme l'indique le titre, de montrer, dans la seconde partie, qu'il existe de tels sens. Nous recourrons à des tests syntagmatiques et paradigmatiques pour prouver le caractère empirique de ces deux propriétés et, partant, pour légitimer de façon non artificielle, la pertinence linguistique du phénomène.

\section{Unité lexicale et pluralité de sens}

\subsection{Observations préliminaires}

On peut accepter comme point de départ provisoire que la polysémie nécessite :

-i- que l'on reconnaisse qu'une forme lexicale (un mot pour aller vite) présente plusieurs sens

-ii- que ces sens soient reliés d'une manière ou d'une autre entre eux.

L'absence de la condition -ii-, qui découle directement de la définition de la polysémie par Bréal (1897) comme étant la conséquence synchronique des changements de sens ${ }^{\mathbf{3}}$, nous met en présence de ce qu'on appelle habituellement l'homonymie.

Une première observation est à faire à ce niveau. On constate dans l'abondante littérature sur la multiplicité des interprétations une intéressante différence entre le traitement de l'homonymie et celui de la polysémie. Si les phénomènes polysémiques sont bien souvent remis en cause, les cas d'homonymie se trouvent généralement épargnés, même si comme pour voler ou grève il s'agit à l'origine d'un réel changement de sens ${ }^{4}$. L'affaire peut sembler anodine. Elle l'est toutefois beaucoup moins, si l'on prend 
en compte le fait que ce qui pousse à nier les faits de polysémie devrait aussi, en grande partie, servir pour remettre en cause les faits d'homonymie. C'est la position à laquelle est amené tout logiquement, dans une perspective cognitiviste, le Cruse de 2004 (Croft et Cruse, 2004, 109) ( $^{\mathbf{2}}$ 《 bounded sense units are not a property of lexical items as such; rather they are construed at the moment of use ». Et un mot homonymique comme bank, dans une vision constructiviste du sens, ne se divisera plus immédiatement en bank-'rive' et bank-'établissement financier', mais se verra associer un seul purport ou matériau conceptuel qui représente l'apport sémantique du mot à la construction de l'interprétation, les sens de 'rive' et de 'banque' étant eux-mêmes des unités de sens construites à partir du «purport» et de conventions $^{6}$. On peut se demander alors pourquoi ceux qui s'en prennent à la polysémie n'attaquent pas avec la même vigueur l'homonymie. La raison, à notre avis, réside dans la présence de la condition -ii- : l'existence de liens (de relations) entre les différents sens d'une même unité lexicale est un facteur qui prédispose à et qui donc favorise la recherche d'une unité sémantique supérieure dégradant les différents sens relevés au rang de simples interprétations de l'aval discursif. Si, par contre, l'écart entre les différentes interprétations n'est pas perçu ou donné comme sous-tendu par un lien, on ne sent pas poussé ou autorisé à chercher un amont unificateur qui déferait la situation polysémique en réduisant ces différentes interprétations à des effets de l'amont en question. .

Une deuxième observation concerne le niveau où s'exerce la critique du fait polysémique. Si on part de la conjonction définitionnelle provisoire -i- et -ii-, la polysémie peut être remise en cause, soit en critiquant -i-, soit en critiquant - ii- $^{7}$. Dans le premier cas, celui où -i- est faux, mais où -ii- subsiste, les relations de -ii- sont à porter au crédit de la construction de lectures qui ne sont plus intrinsèquement attachées à l'expression : on refuse la polysémie, mais on conserve les emplois. C'est la position critique classique. La seconde position, celle où l'on conserve -i-, mais où l'on refuse -ii-, revient à transformer un cas de polysémie en un cas d'homonymie. Elle est, c'est significatif, beaucoup moins fréquente, quoique tout à fait envisageable, comme en témoignent les variations lexicographiques en ce domaine. On peut fort bien s'interroger, par exemple, sur la persistance ou non d'une relation synchronique entre le sens de blaireau-'animal' et blaireau-'brosse pour la barbe'.

Nos deux observations tirent dans la même direction : elles montrent que c'est avant tout le point -i-, celui de la pluralité du sens assignée à une même forme, qui se révèle primordial. Autrement dit, la question essentielle n'est pas tellement celle de l'existence ou non de relations — problématique qui est incontestablement intéressante à un autre point de vue et à un autre niveau, celui des changements de sens et des universaux qui peuvent être impliqués, des potentialités organisationnelles ${ }^{8}$ — mais celle de l'existence multiple de sens associés à une forme, qu'il s'agisse d'homonymie ou de polysémie, même si nous nous plaçons volontairement, étant donné notre première observation, dans le cadre des vocables qui peuvent prétendre concourir pour le label « polysémique ». C'est à ce niveau-là qu'il faut alors poser la question fondamentale : est-ce qu'on peut associer à une unité lexicale plusieurs sens ou non ? Autrement dit encore : est-ce une propriété des unités lexicales, soulignons bien le terme unité lexicale pour éviter toute équivoque, que de pouvoir posséder plusieurs sens ou non ? Est-il justifié d'attribuer à plateau, par exemple, entre autres, le sens de plateau-'vaisselle ' et le sens de plateau-'géographique'? Dit encore autrement, la pratique des lexicographes qui accordent à une unité lexicale plusieurs sens est-elle fondée ou non ? Ces sens sont-il vraiment accrochés à l'unité lexicale ou n'ont-ils qu'une existence discursive, ne sont-ils qu'un produit de la construction du message?

\subsection{Deux manières de nier la polysémie}

Une réponse possible est de refuser d'attribuer à toute unité lexicale du sens préétabli (voir Kleiber, 1999). Le problème de la pluralité de sens se trouve alors réglé, puisque l'exclusion de toute monosémie entraîne en même temps celle de toute pluralité de sens. Nous n'envisagerons pas ici une telle position, parce qu'elle oblige à prendre position sur le statut et la nature du sens en général (Larsson, 1997 et Kleiber, 1999). 
Si l'on met de côté une telle position extrême, mais tout à fait défendable, la polysémie en tant qu'association de plusieurs sens à une même forme lexicale se trouve niée de deux manières apparemment paradoxales :

-a- D’une part, les vocables donnés comme polysémiques se voient en quelque sorte « monosémisés » par la mise en avant d'un invariant supérieur, de quelque nature qu'il soit ${ }^{9}$, qui unifie les différentes interprétations. L'idée, implicite ou explicite, est que ce sens subsumant, explicatif de la diversité interprétative, ne correspond pas aux interprétations observables en discours et que l'on n'y accède qu'en reléguant les sens « immédiats » (surtout les valeurs dénominatives et référentielles) à un étage inférieur, qui n'est plus celui de la langue. Le locuteur lambda n'a évidemment pas accès à ces sens abstraits unificateurs (re)construits grâce à l'observation patiente et sagace du linguiste et qui ont l'avantage de s'insérer dans une conception plus générale de la langue vue comme le passage de formes, de schèmes, de structures abstraites à une incarnation discursive faisant émerger les valeurs, les catégories faussement traitées comme sens premiers par la tradition lexicale et lexicographique. On étend ainsi à toute unité lexicale des concepts comme ceux d'actualisation, de sous-détermination ou de sous-spécification généralement ${ }^{10}$ appliqués au domaine des unités grammaticales, où, comme de nombreuses études l'ont montré, la recherche d'un invariant sous-déterminé et donc le refus d'un éventail trop grand de sens morcelés et non justifiés est tout à fait indiquée ${ }^{11}$. De façon plus générale, empressons-nous de le dire pour éviter toute équivoque sur notre position, la quête d'un amont sémantique pour rendre compte de la diversité des « emplois » d'une unité lexicale est une opération légitime, qui permet, s'il y a effectivement un tel invariant, de débusquer les vocables faussement labellisés polysémiques. Ce qui nous semble, par contre, beaucoup moins légitime, c'est de généraliser la chose et de postuler qu'il en va ainsi de toute unité lexicale, la polysémie alors n'existant plus du tout.

-b- D'autre part, de façon tout à fait inverse aux tentatives de monosémisation, on fait proliférer les sens ou variations de sens. Autrement dit, on procède à la multiplication des cas de polysémie. Soit en postulant qu'il s'agit d'une variation qui n'est pas liée à un lexème particulier, mais qui concerne une série beaucoup plus grande d'unités, parce qu'elle dépend d'une règle ou fonction générale (cf. 'animal' $\longrightarrow$ 'viande de cet animal' pour j'ai mangé du veau) ${ }^{12}$, que l'on appellera polysémie régulière (Apresjan, 1974) ou systématique (Nunberg, 1995, Nunberg et Zaenen, 1997 et Apresjan, 2000)) ou encore polysémie logique (Pustejovsky, 1995) ${ }^{13}$ et dont la généralité implique l'abandon d'un traitement au niveau de l'unité lexicale et, partant, l'abandon de la polysémie comme pluralité sémantique associée à une unité lexicale. Soit surtout en multipliant les variations interprétatives contextuelles pour montrer qu'il ne peut s'agir d'un phénomène du niveau de l'unité lexicale, mais d'un phénomène discursif, lié au contexte, de lectures de circonstance et non de sens du lexème. C'est ainsi que Kayser (1987) assigne à livre l'interprétation 'objet' (manuscrit, disquette) dans l'énoncé Jean écrit un livre, celle d'idées contenues dans ce livre lorsqu'il se combine a influencer quelqu'un (cf. Ce livre a fortement influencé les Révolutionnaires de 1789), celle de la commercialisation dans Ce livre a été un fiasco pour l'éditeur, etc. ${ }^{14}$ Deux résultats découlent d'une telle multiplication: l'impossibilité d'énumérer toutes les interprétations possibles, parce qu'il n'est pas toujours facile de séparer ou de distinguer les différentes interprétations et le renoncement à compter ces différentes interprétations comme des sens du mot, puisqu'ils sont construits par le contexte et donc non autonomes. "Il est artificiel, comme l'écrit Cadiot $(1992,36)$ de leur allouer une existence indépendante de leur émergence ».

Les positions -a- et -b- ne sont qu'apparemment paradoxales : il n'y a aucune contradiction, d'un côté, à faire disparaître la pluralité de sens sous un sens amont ou invariant et, de l'autre, à procéder à la multiplication des petits pains polysémiques, dans la mesure où le point commun des deux positions est que la multiplicité de sens n'est qu'un phénomène discursif et non un phénomène de l'unité lexicale ellemême, puisque dans les deux cas, les sens relevés ne sont pas associés à l'unité lexicale elle-même, mais sont le résultat d'une construction discursive.

A la clef, le verdict sémantique ne fait pas de doute : il n'y a plus lieu de parler de polysémie. Il s'agit d'un jouet fabriqué par les linguistes-sémanticiens, d'un artefact ${ }^{15}$. Les unités lexicales n'ont pas plusieurs sens préétablis, qui leur appartiendraient en propre. Les variations interprétatives présentées ne 
sont que le produit de constructions discursives, où interviennent différents ingrédients, allant jusqu'à la distinction des genres, des textes, etc.

\subsection{Une double condition}

Il serait possible d'examiner et d'évaluer les propositions faites par ceux qui promeuvent une telle approche de toute variation interprétative. Nous ne nous engagerons pas sur cette voie $\mathrm{ici}^{16}$. Il nous semble plus important de nous limiter à la question mise en avant ci-dessus : est-ce qu'il est licite ou non de postuler qu'une unité lexicale (un mot pour aller vite), en tant que telle, peut avoir plusieurs sens ? Est-ce qu'on peut attribuer, de façon non artificielle, à une unité lexicale comme, par exemple, notre plateau de ci-dessus plusieurs sens? Si oui, cela suppose que ces lectures ont émergé des emplois discursifs pour devenir des traits de sens stabilisés et non plus seulement des produits discursifs. Bien évidemment, dire qu'il en va ainsi ne prouve rien. Il revient au linguiste de le démontrer.

A cet effet, il faut qu'il démontre deux choses. Il faut que notre partisan de la polysémie prouve que les acceptions ou lectures des lexèmes auxquels il entend accorder le statut de polysème (et uniquement, bien entendu, à ceux-là) sont bien des sens disjoints, non réductibles, en ce qu'ils ne sont pas « coiffables » par un sens supérieur qui n'en ferait que de simples effets de sens ou de simples emplois issus de la combinaison de ce sens amont avec les différents ingrédients contextuels. L'affaire n'est pas mince, étant donné qu'il semble toujours possible de trouver un chapeau sémantique assez généreux pour accueillir toutes les différenciations interprétatives manifestées par une unité lexicale. Elle n'est toutefois pas impossible, comme on le verra ci-dessous.

Il faut ensuite prouver que ces sens différents disjoints ou séparés non "coiffables » unitairement sont réellement des sens stables accrochés à l'unité lexicale et non simplement des variations sémantiques dues au contexte ou à la construction discursive. Autrement dit, il faut montrer cette fois-ci, non plus qu'il n'y a pas de sens supérieur qui coiffe la diversité interprétative manifestée, mais que les différentes interprétations postulées pour un même lexème sont véritablement des unités sémantiques associées au lexème même et non plus seulement le produit de configurations discursives. Autrement dit, il faut d'une manière ou d'une autre montrer que ces sens n'existent pas seulement discursivement, mais qu'ils ont une forme d'existence lexicale, parce qu'ils ont acquis en quelque sorte une forme de liberté vis-à-vis du contexte ou des constructions dans lesquelles ils se manifestent. C'est prouver en somme que la polysémie est bien une relation lexicale, paradigmatique, ayant acquis sa pertinence au niveau paradigmatique du lexique, parce que les sens en question ont acquis une saillance qui les fait survivre au contexte qui les a fait naître. Il s'agira donc de faire le tri entre les lectures qui restent dépendantes des « circonstances » et celles qui ont gagné leur autonomie, bref, entre celles qui n'arrivent pas à se détacher par rapport à la situation discursive et celles qui montrent une robustesse qui les fait survivre au contexte de leur émergence.

En résumé, les candidats au statut de sens polysémiques, c'est-à-dire de sens associé à une unité lexicale, doivent présenter deux propriétés : ils doivent être non unifiables ou irréductibles à un sens ou lecture générale supérieure et ils doivent en même temps être suffisamment robustes ou forts pour acquérir un statut d'autonomie, qui les détache des circonstances discursives et les sépare des lectures « fragiles» contextuelles, et qui leur permet d'émerger et d'émarger en tant que propriété sémantique stable des unités lexicales. Comment reconnaître qu'ils possèdent ces deux propriétés ? C'est ce que nous essaierons de montrer dans notre deuxième partie.

\section{La polysémie existe, on peut la rencontrer}

\subsection{De nouveau quelques précisions préliminaires}

L'hypothèse qui sous-tend notre démarche est que s'il y a véritablement des sens qui présentent les deux propriétés nécessaires pour que ces sens soient véritablement associés à une unité lexicale et donc 
puissent être qualifiés véritablement de sens polysémiques, ces deux propriétés, c'est-à-dire la nonunifiabilité (ou différence irréductible) et leur robustesse ou autonomie vis-à-vis du discours doivent se manifester de différentes manières, et notamment au niveau de leurs relations syntagmatiques et paradigmatiques où nous retrouverons des tests plus ou moins classiques dans la littérature sur la multiplicité du sens et l'ambiguïté ${ }^{17}$. C'est uniquement l'existence de telles manifestations qui fondent leur pertinence linguistique au niveau lexical et qui assurent à la polysémie ainsi démontrée un statut qui ne peut en rester au stade d'artefact de sémanticien.

On soulignera encore que la possession d'une de ces deux propriétés n'est bien entendu pas suffisante : chaque condition est nécessaire, mais seule elle ne suffit pas, comme nous le verrons. Il faut la conjonction des deux pour conclure à la polysémie.

Troisième précision : il est clair que l'on ne saurait parler de non-unifiabilité que si et seulement s'il y a eu reconnaissance d'une différenciation préalable. Comme nous l'avons montré longuement dans Kleiber (2005), il n'y a généralement pas de discussion sur la multiplicité ou non de sens d'une forme s'il n'y a pas au préalable l'intuition que cette forme peut présenter des interprétations ou des usages différents. Comme cette variation interprétative s'appréhende, qu'on le veuille ou non, avant tout en termes de " choses dénotées ", c'est le sentiment qu'il ne s'agit plus ou pas de la même chose qui est à l'origine de la problématique du sens multiple. Nous avons appelé cette condition de départ (nécessaire, mais bien entendu non suffisante) le critère référentiel (Kleiber, 2005). Nous avons montré, entre autres ${ }^{18}$, que, plus l'autonomie du type d'entité était grande (cf. par exemple les entités exprimées par les substantifs non prédicatifs) et plus il était facile de s'appuyer sur la notion de type de référents différents ou de catégorie différente (ou encore de choses différentes) pour engager une reconnaissance polysémique, alors que, inversement, plus l'entité en question était dépendante et plus délicat était le débat polysémique.

\subsection{Première propriété : non-unifiabilité ou différenciation irréductible}

\subsubsection{Différents niveaux}

La propriété de non-unifiabilité, c'est-à-dire l'impossibilité d'unifier différentes lectures d'une unité lexicale, se manifeste à plusieurs niveaux et se décline sous plusieurs formes, dont certaines sont, bien entendu, plus pertinentes que d'autres.

-1- non-unifiabilité catégorielle : il est difficile, voire impossible, lorsqu'il s'agit de lectures engageant des catégories, de les réduire à une seule catégorie subsumatrice (Kleiber, 2002 et 2004). C'est ainsi qu'il est difficile de concevoir une seule catégorie correspondant aux deux lectures de souris (souris-'animal' et souris d'ordinateur). Corollairement, il est également difficile de trouver un prototype qui correspondrait aux deux lectures, chaque lecture, mais non leur union, donnant lieu à un prototype. La variation interprétative de type vague ou sens sous-déterminé (ou encore sens général), par contre, se révèle de ce point de vue parfaitement unifiable. Même si enfant peut donner lieu aux lectures différentes et incompatibles ${ }^{19}$ 'garçon' et 'fille', il est possible de faire correspondre une catégorie (avec son prototype) qui subsume les catégories 'garçon' et'fille', à savoir la catégorie 'enfant'.

-2- non-unifiabilité vériconditionnelle : l'attribution d'une valeur de vérité s'établit au niveau de chaque lecture particulière et non au niveau d'une interprétation rassemblant les différentes lectures. La différence avec les situations de sens sous-déterminé apparaît ici avec la négation ${ }^{20}$. La proposition Paul n'a pas d'enfant est fausse si l'une des deux lectures (Paul a un garçon / Paul a une fille), incompatibles entre elles, est vraie, alors que Paul n'a pas de souris n'est pas rendue fausse si l'une des deux lectures (souris-'animal' et souris d'ordinateur), incompatibles entre elles, elles aussi, est vraie, parce que la valeur de vérité se décide au niveau de chaque lecture particulière et n'est pas décidable plus haut. Donc même si Paul a une souris d'ordinateur, Paul n'a pas de souris reste vraie s'il s'agit de souris-'animal' et si Paul ne possède effectivement pas de souris-'animal'. Il s'ensuit au niveau discursif, une propriété d'autonomie que Cruse (2000 et 2003) appelle la possibilité de négation indépendante. Il est en effet possible de nier de façon indépendante une des lectures (Cruse, 2000 et 2003) dans le cas de la pluralité 
de sens du type souris, mais non dans le cas des variations interprétatives relevant du vague. A la question $A s$-tu un enfant?, on ne peut répondre non, si l'on a un garçon ou une fille, alors qu'à la question $A s$ - $t u$ une souris ?, il est possible de dire non alors qu'on possède une souris-'animal', si la question porte sur une souris d'ordinateur (et inversement).

-3- non unifiabilité de lectures ${ }^{21}$ : lorsque deux lectures sont en compétition dans un même énoncé, situation d'ambiguïté classique, il n'est pas possible cognitivement d'unifier les deux lectures, comme l'illustrent sur le plan de la perception visuelle les fameuses images ambiguës de la Gestaltthéorie : ou c'est l'une ou c'est l'autre, mais il n'est pas possible de saisir les deux simultanément. Cette nonunifiabilité psychologique permet de montrer que le classique critère d'ambiguïté, appelé critère d'interprétation multiple (Mel'Cuk, Clas et Polguère, 1995, 61), n'est pas suffisant ${ }^{22}$. Le critère d'ambiguïté stipule en effet que si une phrase a deux interprétations différentes dues uniquement à une unité lexicale L, alors L aura deux sens. Or, les unités lexicales de sens vague ou sous-déterminé peuvent répondre à ce critère : Paul a un enfant donne lieu à la variation 'garçon' ou 'fille' et ces deux interprétations sont bien dues à enfant. On ne parlera toutefois pas d'ambiguïté, parce que les deux lectures sont parfaitement unifiables attentionnellement: il n'y a pas de compétition psychologique où l'une doit l'emporter sur l'autre, comme dans le cas de Paul a une souris. Cela apparaît nettement si on oppose Paul voudrait un enfant à Paul voudrait une souris.

-4- non-unifiabilité sémantique : il n'est pas possible de trouver un sens général, plus abstrait, rassemblant et organisant les différentes lectures. C'est un point névralgique, puisque, comme nous l'avons vu, une des deux manières de nier la polysémie, consiste précisément à toujours trouver un sens abstrait explicateur. Ce point est d'autant plus névralgique qu'il est toujours possible de trouver un tel sens «coiffant» en prenant les traits communs à toutes les lectures ou emplois relevés. Il y a toutefois un problème, celui de la pertinence d'un invariant construit de la sorte. Il est bien souvent trop abstrait et par là-même incontrôlable : on oublie généralement de montrer comment se fait la «descente » vers les interprétations attestées ${ }^{23}$. Mais le danger le plus grand est celui d'une trop grande puissance : on trouve, certes, des dénominateurs communs à toutes les lectures relevées, mais ce sens construit à partir des traits communs, s'il se retrouve effectivement toujours dans les lectures particulières, court le risque de présenter un pouvoir prédictif trop fort : il lui arrive en effet de prévoir des sens ou lectures (ou emplois) qui n'existent pas, au point qu'il apparaît dans ce cas comme étant un artefact du linguiste qui l'a construit. Martin $(2005,170)$ cite ainsi le cas de quartier et montre que, quel que soit le sens général supérieur proposé (comme par exemple 'partie d'un tout'), même si ce sens rend compte des différents sens ou emplois recensés (quartier de viande, quartier de ville, quartier des femmes, etc.), il ne pourra pas expliquer pourquoi on n'a pas quartier d'un livre, pour le chapitre d'un livre, ou quartier d'une symphonie pour le mouvement d'une symphonie. «Rien, conclut-il $(2005,170)$, ne permet de le prévoir. La seule solution est d'inscrire les possibles dans le dictionnaire ».

Bref, il existe incontestablement des lectures différentes d'une même forme qui ne sont sémantiquement pas unifiables, de façon non artificielle. Ce ne sont pas, automatiquement, on le verra, des sens polysémiques, mais les sens qui prétendent représenter un fait de polysémie doivent posséder une telle propriété.

\subsubsection{Tests syntagmatiques et paradigmatiques}

\subsubsection{Syntagmatiques}

Il y a des correspondants formels syntagmatiques et paradigmatiques à la non-unifiabilité que nous venons de mettre en avant. Au niveau syntagmatique, on observe en effet que les structures et constructions qui supposent d'une manière ou d'une autre une forme d'unité sont plus ou moins récalcitrantes au sens multiple non unifiable. On retrouve là certains tests bien souvent appelés à la rescousse dans la littérature sur la polysémie et l'ambiguïté. Tous n'ont pas la même pertinence, mais tous tirent dans la même direction. 
En premier lieu, on mentionnera un critère guère utilisé, car pas facilement manipulable et d'application limitée aux SN, mais pourtant intéressant : la difficulté d'avoir pour les sens non unifiables un SN avec une interprétation générique "coiffante », c'est-à-dire recouvrant les différentes lectures. Face aux situations de sens général, où la chose est tout à fait possible, comme le montre un énoncé du type Les enfants sont toujours ingrats, qui recouvre aussi bien la spécification 'garçon' que 'fille', il n'est guère possible d'envisager un énoncé générique similaire avec le SN les souris «couvrant» de la même manière aussi bien les souris-'animaux' que les souris d'ordinateur. Un tel regroupement n'est possible qu'au niveau métalinguistique, celui qui repose sur la pluralité de lectures et il faut que la phrase soit à la forme négative et comporte le quantificateur de totalité, ceci pour rappeler précisément que l'unification n'est pas possible, parce qu'il y a plusieurs types de choses appelées X. C'est ainsi que l'on peut avoir en regroupement : toutes les souris ne sont pas des animaux, mais non pour une union du même type : Les souris ne sont pas des animaux, ni Toutes les souris sont des animaux.

En deuxième lieu, on dispose du critère de cooccurrence compatible (Mel'Cuk, Clas et Polguère, 1995), appelé aussi critère de Green-Apresjan ${ }^{24}$ par par Mel'Cuk, Clas et Polguère ou critère des sens antagonistes par Cruse (1986). (Green 1969 et Apresjan, 1992). Ce critère établit qu'il y a sens multiple si et seulement si la cooccurrence discursive des différentes lectures ou interprétations se révèle impossible, en somme si les différents sens ne sont pas compatibles, unifiables et se révèlent donc antagonistes, disjoints. Il s'agit d'un indicateur assez fiable qui projette sur le plan syntagmatique l'incompatibilité paradigmatique des sens concernés. L'idée qu'il traduit est que, si deux lectures d'une même unité lexicale sont vraiment deux lectures autonomes, non unifiables, elles ne sont pas non plus compatibles en les mettant ensemble au niveau syntagmatique. Ce critère revêt plusieurs formes. La coordination de constituants qui activent les différentes interprétations est souvent utilisée. C'est ainsi que si l'on prend le cas de veau-'animal' et veau-'viande de cet animal', la coordination de deux adjectifs qui activent les deux lectures aboutit à un zeugme ${ }^{25}$, qui révèle bien leur incompatibilité :

\footnotetext{
? Ce veau est rapide et tendre (dans le sens où tendre porte sur la viande)
}

La contrainte d'identité (Cruse, 1986) est une autre forme de ce test syntagmatique. Elle exige qu'en cas d'anaphore du type aussi, tout comme, ainsi fait $X$... ? l'on ne puisse avoir une interprétation croisée, l'antécédent et l'anaphore ne pouvant être de lecture différente. L'énoncé Pierre aime ce plateau, Paul aussi ne connaît ainsi que des interprétations d'identité. S'il s'agit du plateau-'vaisselle' pour Pierre, il en va également ainsi pour Paul et si c'est le plateau géographique, il en va encore ainsi pour Paul. Ce qui se trouve exclu, c'est l'interprétation croisée où, par exemple, pour Pierre il s'agirait du plateau-'vaisselle' et pour Paul du plateau géographique. Si on applique ce test au sens sous-déterminé, on constate que les interprétations croisées sont cette fois-ci permises. L'énoncé Marie attend un enfant, Berthe aussi donne lieu à quatre lectures, deux d'identité (une où Marie et Berthe attendent toutes les deux une fille et une où ils attendent un garçon), mais aussi deux de non identité (une où Marie attend un garçon et Berthe une fille et l'autre où c'est l'inverse). Trouvent leur place également ici les relatives qui en cas de lectures non réductibles ne permettent pas une divergence référentielle entre l'antécédent et le pronom relatif (cf. J'aime faire des promenades sur le plateau qu'a acheté Pierre pour compléter la vaisselle). Il en va de même pour les anaphores réalisées par les pronoms de $3^{\mathrm{e}}$ personne (Ce plateau est très fréquenté. Il est en faïence), qui permettent de rectifier certaines analyses opérées à propos d'un énoncé tel que Georges Sand est sur l'étagère de gauche : contrairement à ce que l'on pourrait attendre, un tel énoncé ne se prête qu'à une anaphore sur l'écrivain et non sur les œuvres (Georges Sand est sur l'étagère de gauche. Tu verras, elle écrit divinement /* Georges Sand est sur l'étagère de gauche. Elle / ils est / sont relié(s) en cuir) (Kleiber, 1994 et 1999) ${ }^{26}$.

Troisièmement, le critère de distribution différente (ou de cooccurrence différentielle selon Mel'Cuk, Clas et Polguère, 1995) est une autre manifestation de la non-unifiabilité. L'idée sous-jacente est que la dissociation des sens se traduit aussi par une dissociation distributionnelle. Ou, autrement dit, la nonunifiabilité des lectures apparaît dans une non unification distributionnelle correspondante : si on peut dégager deux ensembles disjoints de cooccurrents (morphologiques, syntaxiques ou lexicaux) tels que l'un correspond à S1 et l'autre à S2, alors on a sens multiple (Mel'Cuk, Clas et Polguère, 1995). Citons ici le cas classique du verbe regarder souvent utilisé pour prouver l'efficacité du critère de différenciation 
distributionnelle dans la séparation du sens locatif ('être tourné vers') du sens standard perceptuel actif. Les exemples parlent d'eux-mêmes :

\author{
Paul regarde la montagne \\ Paul a regardé la montagne \\ L'église regarde la montagne \\ *L'église a regardé la montagne \\ Ce match a été regardé par toute la population \\ * La montagne a été regardée par l'église \\ Paul a regardé intentionnellement la montagne \\ * L'église regarde intentionnellement la montagne
}

Deux remarques sont nécessaires. La première concerne la diversité des faits syntagmatiques à prendre en compte : constructions, contraintes sélectionnelles, collocations ${ }^{27}$, etc. : tous n'ont pas la même importance et il conviendrait d'établir une hiérarchie pour voir lesquels l'emportent sur d'autres. Deuxièmement, ce critère de distribution différentielle est un des critères auxquels on a recours le plus fréquemment pour prouver les faits de sens multiples, mais il faut souligner que, plutôt qu'un critère de reconnaissance, c'est plus une manifestation de la pertinence d'une pluralité de sens déjà diagnostiquée autrement, qu'elle peut donc venir appuyer ou confirmer, mais non reconnaître elle-même. Nous voulons dire par là que l'utilisation de ce critère à l'aveugle, c'est-à-dire sans délimitations sémantiques déjà effectuées conduit bien vite à des impasses. La raison en est bien simple. On a pour la majorité des sens ou lectures des différences de cooccurrence (dans le sens large indiqué ci-dessus). Si l'on s'en tenait donc uniquement à de telles différences sans contrôle sémantique et si l'on considérait ces différences comme décisives pour la délimitation du sens multiple, il faudrait conclure à autant de sens différents que de phénomènes distributionnels différents. Notre mise au point montre ainsi que ce critère est le plus efficace là où les différences interprétatives sont les plus nettes et s'avère beaucoup plus fragile ou délicat à manipuler là où les hésitations sont permises (y a-t-il différence d'interprétation ou non ?). C'est, dans ce dernier cas, que les analyses peuvent diverger, parce qu'elles se servent de tel ou tel fait de cooccurrence différentielle pour faire basculer le sens vers le multiple ou non.

\title{
3.2.2.2 Paradigmatiques
}

La non-unifiabilité se laisse aussi appréhender au niveau paradigmatique : l'hypothèse est qu'il n'y a pas de relations au niveau paradigmatique qui conduiraient ou induiraient une union des deux unités de sens (ou plus) testées. Les sens disjoints testés présenteraient ainsi des ensembles de relations lexicales paradigmatiques disjointes, en autonomie relationnelle (Croft et Cruse, 2004, 113).

En premier, lieu, l'absence d'hyperonyme pour couvrir lexicalement les deux lectures est un écho direct de la non-unifiabilité sémantique. S'il y a un hyperonyme disponible pour "coiffer» les différentes lectures, on peut en conclure qu'il n'y a pas sens multiple. Le sens général ou vague se trouve ainsi définitoirement éliminé. L'inverse n'est bien entendu pas vrai : l'absence d'hyperonyme ne signifie pas automatiquement qu'il y a sens multiple.

En deuxième lieu, la synonymie (intra- et interlinguistique ${ }^{28}$ ) s'avère être fort utile : le test consiste à montrer que deux lectures non unifiables ont également deux ensembles de synonymes disjoints, non unifiables $^{29}$. On se contentera de citer sans le commenter l'exemple de l'adjectif juste emprunté à Gross $(1998,111)$ :

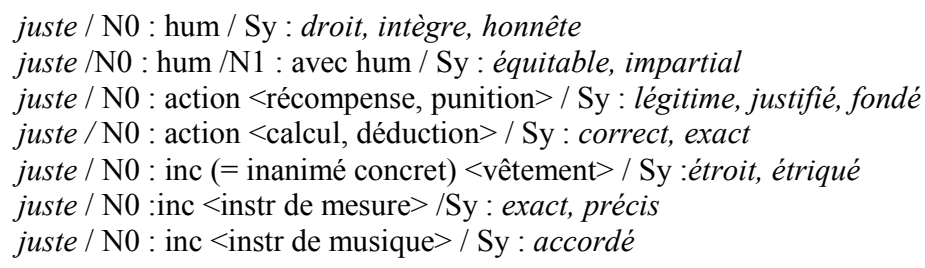

On ajoutera que, tout comme les collocations (cf. supra), la synonymie est aujourd'hui au premier plan dans les affaires polysémiques. Certains vont jusqu'à en faire l'instrument principal d'une approche de la 
polysémi $^{30}$ : «On peut représenter la polysémie (ou dans la terminologie de la sémantique lexicale européenne, le champ sémasiologique) d'une unité lexicale sous la forme d'un espace sémantique présentant autant de dimensions que de synonymes qui lui sont associés » (François, Victorri et Manguin, 2005, 175).

La synonymie appelle tout naturellement l'antonymie : la dissociation de deux lectures peut se manifester également dans l'existence d'antonymes différents, non réductibles à un antonyme supérieur ${ }^{31}:$ si l'on a une valise légère comme un café léger, l'on n'aura pas face à une valise lourde, un *café lourd.

En quatrième lieu, des dérivés différents selon le sens peuvent aussi venir appuyer une dissociation interprétative. (cf. le critère de dérivation différentielle de Mel'Cuk, Clas et Polguère, 1995, 68). Ainsi les trois lectures d'élever dans a), b) et c) donnent lieu à des dérivés différents (exemple de Mel'Cuk, Clas et Poguère, 1995, 68) :

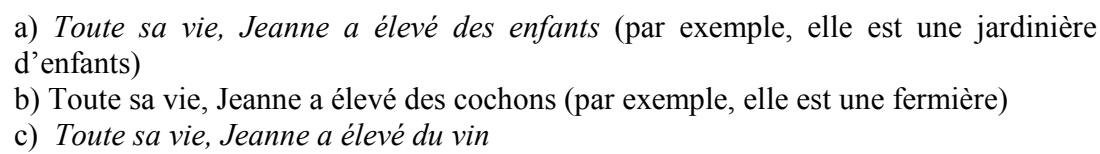

Pour b), il y a le nom d'action dérivé élevage (Jeanne s'occupe d'élevage de cochons), le nom d'agent éleveur / éleveuse (Jeanne est éleveuse de cochons). Pour a) ces dérivations sont impossibles : * élevage d'enfants , *éleveur d'enfants, mais on a des dérivés lexicaux (des supplétifs) : éducation, formation, instituteur, jardinière d'enfants. Pour c), on n'a pas *élevage de vin, mais éleveur de vin.

Il y aurait d'autres pistes à creuser, celle des champs sémantiques notamment, mais pour nous l'essentiel est d'avoir montré la diversité des faits paradigmatiques qui peuvent venir conforter l'existence de sens non unifiables. Il faut se rappeler que ces critères ne sont pas décisifs - Cruse (1986) parle de tests indirects - car leur caractère paradigmatique suppose leur détachement préalable et leur stabilisation par rapport aux configurations syntagmatiques qui les actualisent.

\subsection{Deuxième propriété : « la polysémie prise au mot » ${ }^{32}$}

\subsubsection{Pourquoi les tests de non-unifiabilité ne suffisent pas}

Généralement, on fait comme si les critères de non-unifiabilité suffisaient pour garantir le caractère polysémique des sens différents examinés. Nous avons toutefois bien souligné que la propriété de nonunifiabilité n'était à elle seule pas décisive, parce qu'elle ne garantissait pas que les sens déclarés antagonistes ou irréductibles étaient véritablement des sens associés à l'unité lexicale. Mis à part les tests paradigmatiques, parce qu'ils supposent une stabilisation lexicale hors contexte, beaucoup de tests que nous avons vus s'appliquent à des phénomènes de variation interprétative qui ne relèvent pas de la polysémie parce qu'ils ne possèdent pas la deuxième propriété postulée, à savoir la robustesse ou autonomie par rapport au discours. Dans ses différents travaux (voir bibliographie), Cruse insiste sur ce point en montrant qu'une variation de sens comme celle de la célèbre « omelette » ('plat' et 'client qui a commandé ce plat') :

\section{L'omelette est parti(e) sans payer}

répondait aux critères des sens antagonistes, mais ne pouvait prétendre au statut de polysémie, parce qu'elle restait une lecture $d$ " circonstance ». La vérification de son caractère non unifiable est facile à faire : pas de catégorie ni de sens subsumant possible, pas d'unifiabilité des lectures et peu de tentatives même chez les monosémistes pour trouver un invariant aux deux lectures. Les tests syntagmatiques confirment cette analyse : pas d'interprétation générique « coiffante » possible, cooccurrence discursive des deux lectures impossible (*L'omelette est trop salée et est assise à la troisième table), contrainte d'identité nécessaire (pas de lecture croisée possible pour Pierre a servi l'omelette, Paul aussi, pas de divergence dans une phrase avec relative * L'omelette, qui était trop salée, est parti(e) sans payer), etc. Les tests paradigmatiques, comme déjà annoncé, ne s'y appliquent guère, précisément parce qu'une des 
lectures (celle du 'client') n'est pas reconnue comme étant un sens stabilisé à rattacher en propre à l'unité lexicale omelette.

\subsubsection{Autonomie et pertinence lexicale}

Deux conditions doivent être remplies pour que l'on puisse parler de lectures propres à l'unité lexicale, de lectures qui n'émergent pas seulement au niveau syntagmatique, mais qui ont également acquis un statut paradigmatique. Premièrement, elles doivent bien avoir pour source l'unité lexicale en question et non pas une autre source. En second lieu, elles doivent être assez robustes pour avoir une existence hors discours, au niveau de l'unité lexicale.

La première condition permet d'éliminer deux types de situations de sens multiple : les lectures accrochées aux locutions figées (noms composés compris) et celles qui sont le produit de mécanismes polysémiques plus généraux.

Dans le premier cas, étant donné la non compositionnalité sémantique de l'expression figée, on ne peut reporter sur un constituant une partie ou le sens global de l'ensemble de l'expression. On ne saurait, pour reprendre un exemple classique de la littérature, parler de la multiplicité de sens d'arbre en y faisant entrer la lecture correspondant à arbre à came, parce qu'arbre dans arbre à came n'est pas libre ${ }^{33}$ et donc l'interprétation qu'on associe à arbre à came ne saurait être répercutée sur celle d'arbre. Il s'agit d'une question cruciale, puisqu'une bonne partie des détracteurs de la notion de polysémie, non seulement intègrent ce type d'expressions figées, mais les considèrent comme meilleurs pour l'exploration du sens de l'unité lexicale que les emplois « libres ».

Le second cas a été déjà été abordé ci-dessus. Les polysémies appelées régulières ou systématiques, quel que soit leur degré de généralité, ont pour caractéristique de ne pas être dépendantes de l'unité lexicale précise qui les actualise, mais de grandes règles (cognitives ou autres). Il s'ensuit que, comme l'ont souligné tous ceux qui ont mis en avant ce type de transferts sémantiques, on ne saurait porter les lectures obtenues par ces règles au crédit des unités lexicales qui les manifestent. Reprenons ici le cas de l'omelette qui est partie sans payer: le transfert métonymique s'applique à tout plat commandé et ne concerne donc pas directement le lexème omelette et, partant, interdit d'associer à omelette directement le sens de 'client qui a commandé une omelette'. En abordant la deuxième condition on verra qu'il y a une deuxième raison à ne pas effectuer un tel choix.

La deuxième condition impose le détachement vis-à-vis du discours, l'autonomie vis-à-vis du contexte ou de la situation, propriété qui permet de parler de la robustesse ou de la force des lectures puisque celles-ci subsistent dans ce cas aux situations discursives qui les ont fait émerger. Qu'on ne se méprenne pas : cela ne veut pas dire que la compréhension ne nécessite pas une situation discursive précise (et que donc la levée d'ambiguïé possible ne s'opère pas par le contexte). Ce qu'il faut entendre par là, c'est uniquement que les lectures ont acquis assez d'indépendance pour apparaître comme une propriété du lexème et non plus seulement comme la conséquence d'une construction discursive. Autrement dit, les lectures qui prétendent au statut de polysémie ne peuvent pas être seulement des lectures de circonstance, qui ne survivent pas à la situation qui les a produites, mais doivent avoir gagné leur pertinence au niveau même de l'unité lexicale.

Se trouvent exclus en conséquence tous les emplois « figurés » circonstanciels (cf. métaphores vives et autres tropes créatifs), qui ne sont pas stabilisés au niveau de l'unité lexicale ${ }^{34}$. La chose n'a rien d'étonnant, étant donné que ce type de décalage sémantique « rhétorique » n'est généralement pas retenu dans la problématique de la polysémie.

Plus intéressants par contre se révèlent les transferts de sens plus ou moins réguliers déjà entrevus cidessus, lors de la première condition, parce que ce sont des candidats sérieux au statut de polysémie et qu'ils permettent de mieux voir en quoi consiste la question de l'autonomie ou non-autonomie circonstancielle, de la robustesse ou non des lectures en jeu. Soit une nouvelle fois donc notre exemple de l'omelette partie sans payer. Qu'il s'agit d'une lecture non robuste, non autonome, transparaît à trois niveaux : situationnel, syntagmatique et paradigmatique. 
Au premier niveau, on note une limitation situationnelle très forte, signalée par Cruse (2003). Il semble difficile de pouvoir utiliser, sans faire sourire (on retombe alors sur le cas des emplois figurés créatifs), le SN l'omelette pour le client en dehors de la situation que représente le restaurant avec ses clients et ses serveurs (ou une autre situation impliquant des plats). De façon plus précise encore, il faut que celui qui utilise l'expression l'omelette ainsi soit dans une situation qui légitime une telle utilisation. On comprend pourquoi le serveur puisse s'en ...servir (!) : le client existe pour lui avant tout par l'intermédiaire du plat commandé. Il est beaucoup plus difficile, mais non impossible, qu'une autre personne présente dans le restaurant désigne ainsi l'omelette en question. On pourrait citer ici bien d'autres cas, comme celui de $\mathrm{Je}$ suis garé pas loin d'ici (Kleiber, 1995 et 1999) qui ne peut se concevoir que dans la situation où le locuteur est effectivement «automobiliste ». S'il est assis dans son salon en train de regarder la télé, il ne pourra dire : je suis garé dans mon garage, même si sa voiture est effectivement garée dans son garage. De même on ne peut avoir Schumacher perd de l'huile que s'il est en train de rouler en voiture.

$\mathrm{Au}$ deuxième niveau, on observe que les prédicats qui accompagnent les expressions décalées sont beaucoup plus restreints que l'aire prédicative délimitée par le sens du référent désigné. C'est ainsi que George Sand peut être sur l'étagère de gauche (Georges Sand est sur l'étagère de gauche), mais on ne saurait avoir, comme on pourrait l'attendre de la sélection opérée par le sens 'les œuvres ou livres de l'écrivain ainsi nommé', Georges Sand est tombé par terre (même dans la situation où les livres de Georges Sand de l'étagère en question sont tombés par terre). On notera inversement - ce qui est révélateur également — que ce sens d'omelette n'a quasiment pas de collocations spécifiques comme les lectures multiples à statut polysémique.

$\mathrm{Au}$ troisième niveau, enfin, toutes ces lectures n'ont pas accès aux relations paradigmatiques, qui marquent précisément par détachement syntagmatique l'accessibilité au statut paradigmatique de sens lexical, c'est-à-dire son rattachement à l'unité lexicale. Cruse (2003) a fort bien noté ce manque de robustesse à propos de l'exemple de l'omelette. Même si les sens 'plat' et 'client qui a commandé ce plat' sont non unifiables, comme nous l'avons vu, la lecture 'client qui a commandé ce plat ${ }^{35}$ n'accède pas au niveau des relations sémantiques qui structurent le lexique. Il n'a pas acquis le statut d'hyponyme de 'client' et donc client n'est pas son hyperonyme (? Les omelettes et autres clients) et, contrairement aux autres exemples de sens multiple que nous avons mentionnés ci-dessus il n'entre pas dans des réseaux de synonymie, d'antonymie et ne donne pas lieu à des dérivés spécifiques.

\section{Conclusion}

Il existe donc bien, ce sera notre conclusion, des variations interprétatives qui ont des propriétés particulières, la non-unifiabilité et une autonomie discursive, qui se traduisent, comme nous avons essayé de le montrer, par des manifestations syntagmatiques et paradigmatiques spécifiques. On ne saurait nier ces manifestations linguistiques : si pour telle situation de sens multiple une anaphore ne peut s'établir ou s'il n'est pas possible de coordonner des constituants qui activent les différentes interprétations sans effet de zeugme ou si encore telle lecture connait des relations paradigmatiques que telle autre ne connaît pas, c'est que ces propriétés sont des propriétés empiriques, qui prouvent in fine qu'il ne s'agit pas d'un artefact dû à une tradition logico-grammaticale qui a toujours bon dos ou à je ne sais quel autre commode épouvantail théorique que l'on agite avec une opacifiante gourmandise non dissimulée. Et, partant, ces données prouvent aussi que la mise en avant de variations interprétatives qui présentent la conjonction des deux propriétés n'est pas non plus artificielle. Il ne s'agit, bien entendu, pas encore de polysémie, puisque celle-ci nécessite en plus une analyse interne des lectures qui mette au jour la ou les relations unissant ces lectures, point que nous n'avons pas abordé ici. Et on peut, si on le désire, refuser le terme de polysémie et celui d'homonymie. Ce n'est après tout qu'une affaire d'étiquettes. Mais ce que l'on ne saurait refuser, c'est que certaines interprétations multiples ont des propriétés empiriques particulières qui font qu'elles sont linguistiquement pertinentes au niveau de l'unité lexicale elle-même et non plus seulement du discours. C'est ce point, souvent nié aujourd'hui, qui me semble essentiel. Pour le dire autrement, si en matière de polysémie tous les sens ne sont pas permis, la polysémie elle-même n'est pas un sens interdit. 


\section{Références bibliographiques}

Apresjan, J., 1974, Regular Polysemy, Linguistics, 142, 5-32.

Apresjan, J, 1992, Lexical Semantics, Ann Arbor, Karoma Publisher.

Apresjan, J., 2000, Systematic Lexicography, Ann Arbor, Karoma Publisher.

Barque, L., 2008, Description et formalisation de la polyséie régulière du français, Thèse de Doctorat, Université de ParrisX.

Blank, A., 2005, Polysemie : Sprachbewusstsein, semantische Relationen, Diskurstraditionen, in Jacob, D., Krefeeld, T et Oesterreicher, W. (hrsg.), Sprache, Bewusstsein, Stil. Theoretische und historische Perspektiven, Tübingen, Gunter Narr Verlag, 31-46.

Blumenthal, P. et Hausmann, F.J. (éds), 2006, Collocations, corpus, dictionnaires, ${ }^{\circ} 150$ de Langue française.

Bréal, M., 1897, Essai de sémantique, Paris, Hachette.

Bres, J., à paraître, La polysémie à l'épreuve des temps verbaux : actualisation, interaction, effets de sens produits.

Cadiot, P., 1992, Extensions et glissements polysémiques ... d'une langue à l'autre, in Lorgnet, M. (éd.), Atti della fiera Internazionale delle Traduzione, Forli, Ateneo Editrice, vol. 1, 31-56.

Cadiot, P. et Visetti, Y.-M., 2001, Pour une théorie des formes sémantiques. Motifs, profils, thèmes, Paris, PUF.

Constantin de Chanay, H. et Remi-Giraud, à paraître, La polysémie prise au mot? Construction du sens et polysémioticité.

Croft, W. et D.A. Cruse, 2004, Cognitive Linguistics, Cambridge, Cambridge University Press.

Cruse, D.A., 1986, Lexical Semantics, Cambridge, Cambridge University Press.

Cruse, D.A., 1995, Polysemy and related phenomena, in St. Dizier, P. et Viegas, E. (eds), Computational Lexical Semantics, Cambridge, Cambridge University Press, 33-49.

Cruse, D.A., 1996, La signification des noms propres de pays en anglais, in Rémi-Giraud, S. et Rétat, P. (éds), Les mots de la nation, Lyon, Presses Universitaires de Lyon, 93-102.

Cruse, D.A., 2000, Aspects of the Micro-structure of Word Meanings, in Ravin, Y. et Leacock, C. (eds), Polysemy. Theoretical and Computational Approaches, New-York, Oxford University Press, 30-51.

Cruse, D.A., 2003, Aux frontières de la polysémie : les micro-sens, in Rémi-Giraud, S. et Panier, L. (éds), La polysémie ou l'empire des sens. Lexique, discours, représentations, Lyon, Presses Universitaires de Lyon, 131140.

Franckel, J.J., 2006, Situation, contexte et valeur référentielle, Pratiques, n¹29-130, 51-70.

François, J., 2007, Pour une cartographie dela polysémie verbale, Leuven-Paris, Editions Peeters.

François, J., Victorri, B. et Manguin, J.-L., 2005, Polysémie adjectivale et synonymie, in Soutet, O. (éd.), La polysémie, Paris, Presses de l'Université Paris-Sorbonne, 175-188.

Green, G., 1969, On the Notion 'Related Lexical Entry', Papers from the Fifth Regional Meeting, Chicago, Chicago Linguistic Society, 76-88.

Gross, G., 1998, Pour une véritable fonction "synonymie" dans un traitement de texte, Langages, 131, 103-114.

Kayser, D., 1987, Une sémantique qui n'a pas de sens, Langages, 87, 33-45.

Kleiber, G., 1990, Sur la définition sémantique d'un mot: les sens uniques conduisent-ils à des impasses ?, in Chaurand, J. et Mazière, F. (éds), La définition, Paris, Larousse, 125-148.

Kleiber, G., 1994, Nominales. Essais de sémantique référentielle, Paris, Armand Colin.

Kleiber, G., 1995, Polysémie, transferts de sens et métonymie intégrée, Folia Linguistica, XXIX, 1-2, 105-132.

Kleiber, G., 1996, Cognition, sémantique et facettes : une histoire de livres et de ... romans, in Kleiber, G. et Riegel, M. (éds), Les formes du sens. Etudes de linguistique française médiévale et générale offertes à Robert Martin à l'occasion de ses 60 ans, Louvain-la-Neuve, Duculot, 219-231. 
Kleiber, G., 1998, Est-ce qu'un veau peut être rapide et tendre ?, Romanische Forschungen, 110, 327-347.

Kleiber, G., 1999, Problèmes de sémantique. La polysémie en questions, Villeneuve d'Ascq, Presses du Septentrion.

Kleiber, G., 2000, A l'ECOLE de la polysémie, in Buvet, P.-A., Colas-Matthieu, M. et Lepesant, D. (éds), Lexique, Syntaxe et Sémantique, Mélanges offerts à Gaston Gross à l'occasion de son 60e anniversaire, Numéro HorsSérie BULAG, Centre Tesnière Besançon, 391-408.

Kleiber, G., 2002, De la polysémie en général à la polysémie prototypique en particulier, Cahiers de lexicologie, 80 : $1,89-103$.

Kleiber, G., 2004, Polysémie et catégories, Verbum, XXVI : 1, 100-117.

Kleiber, G., 2005, Quand y a-t-il sens multiple ? Le critère référentiel en question, in Soutet, O. (éd.), La polysémie, Paris, Presses de l'Université Paris-Sorbonne, 51-73.

Larsson, B., 1997, Le bon sens commun. Remarques sur la (re)cognition intersubjective dans l'épistémologie et l'ontologie du sens, Lund, Lund University Press.

Martin, R., 1972, Esquisse d'une analyse formelle de la polysémie, Travaux de linguistique et de littérature, 10, 125136.

Martin, R., 1979, La polysémie verbale, esquisse d'une typologie formelle, Travaux de linguistique et de littérature, $17,251-261$.

Martin, R., 2001, Sémantique et automate, Paris, PUF.

Martin, R., 2005, Traitement automatique de la polysémie. Eloge du dictionnaire, in Soutet, O. (éd.), La polysémie, Paris, Presses de l’Université Paris-Sorbonne, 167-173.

Mel'Cuk, I., Clas, A. et Polguère, A., 1995, Introduction à la lexicologie explicative et combinatoire, Louvain-laNeuve, Duculot.

Nunberg, G., 1995, Transfers of Meaning, Journal of Semantics, 17, 109-132.

Nunberg, G. et Zaenen, A., 1997, La polysémie systématique dans la description lexicale, Langue française, 113, 1223.

Picoche, J. et Rolland, J.C., 2002, Dictionnaire du français usuel, Bruxelles, De Boeck et Duculot.

Pustejovsky, J., 1995, The Generative Lexicon, Cambridge, MIT Press.

Rastier, F., Cavazza, M. et Abeillé, A., 1994, Sémantique pour l'analyse. De la linguistique à l'informatique, Paris, Masson.

Saulnier, E., 1996, Identité lexicale et régulation de la variation sémantique. Contribution à l'étude des emplois de mettre, prendre, passer et tenir, Thèse de Doctorat, Nanterre, Université de Paris X.

Wierzbicka, A., 1985, Lexicography and Conceptual Analysis, Ann Arbor, Karoma Publishers.

\footnotetext{
${ }^{1}$ Le titre prévu à l'origine était : La polysémie : une affaire vraiment ... ambiguë.

${ }^{2}$ Ou homonymique (voir nos observations préliminaires).

3 «Le sens nouveau, quel qu'il soit, ne met pas fin à l'ancien. Ils existent tous les deux l'un à côté de l'autre. Le même terme peut s'employer tour à tour au sens propre ou au sens métaphorique, au sens restreint ou au sens étendu, au sens abstrait ou au sens concret...A mesure qu'une signification nouvelle est donnée au mot, il a l'air de se multiplier et de produire des exemplaires nouveaux, semblables de forme, mais différents de valeur. Nous appelons ce phénomène de multiplication la polysémie (de polus, "nombreux", et sèmeion, "signification") 》 (Bréal, 1897, 143-144).

${ }^{4}$ Que voler-'dérober' soit issu de voler-'se déplacer dans les airs' par l'intermédiaire de 'chasser en volant' (ancien français) n'est pas admis par tout le monde.

${ }^{5}$ C'est Cruse qui est l'auteur du chapitre sur la polysémie.
} 
${ }^{6}$ « Each lexical item (word form) is associated with a body of conceptual content that is here given the name purport. Purport is part of the raw material contributed by the word to processes of construal of an interpretation (the other part being a set of conventional constraints. » (Croft et Cruse, 2004, 100).

${ }^{7}$ On peut aussi remettre les deux en cause simultanément : l'homonymie disparaît dans ce cas-là également.

${ }^{8}$ Voir à cet égard Martin (1972 et 1979), Picoche et Rolland (2002), Blank (2005), etc.

${ }^{9}$ Voir le courant de Cadiot et Visetti (2001) et celui de Franckel (2006).

${ }^{10}$ Elle correspond aussi aux lexèmes qui donnent lieu au vague ou sens général, que nous aborderons ci-dessous.

${ }^{11}$ Voir Bres (à paraître) qui met « la polysémie à l'épreuve des temps verbaux ».

${ }^{12}$ Cf . Kleiber (1998 et 1999).

${ }^{13}$ Barque (2008) montre fort clairement qu'il n'y a pas équivalence.

${ }^{14}$ Voir le débat que nous avons eu avec Kayser à ce propos autour de 1990 (Kleiber, 1990).

15 «Les problèmes de la polysémie et de l'ambiguïté qui préoccupent la sémantique sont pour l'essentiel des artefacts de la conception essentialiste de la signification » (Rastier, Cavazza et Abeillé, 1994, 51).

${ }^{16}$ Nous l'avons fait à plusieurs reprises ailleurs (voir bibliographie).

${ }^{17}$ Exprimons notre dette vis-à-vis de Cruse (1986, 1995, 1996, 2000, 2003 ; Croft et Cruse, 2004), de Mel' Cuk, Clas et Polguère (1995) et Martin (2001 et 2005).

${ }^{18}$ Voir Kleiber (2005) pour d'autres conclusions en relation directe avec la variation référentielle.

${ }^{19} \mathrm{Si} \mathrm{x}$ est un garçon, ce ne peut être une fille.

${ }^{20}$ Elle ne se manifeste pas avec le positif (cf. Paul a un enfant / Paul a une souris).

${ }^{21}$ Cruse (dans Croft et Cruse, 2004) parle à ce propos d'autonomie attentionnelle.

${ }^{22}$ Comme le soulignent d'ailleurs Mel'Cuk, Clas et Polguère, puisqu'ils ajoutent qu'une indication contraire (donnée par un autre critère) le rend caduque.

${ }^{23}$ Voir Kleiber (1999). Mentionnons uniquement ici la définition de tenir donnée par Saulnier (1996) et rapportée par Franckel $(2006,60)$ : «tenir marque qu'étant donné une relation de référence R et une instabilité (R,R') telle que "autre que R" est envisageable, un terme est source de la validation de $\mathrm{R}$ ».

${ }^{24}$ Il s'agit de Green (1969) et d'Apresjan (1992).

${ }^{25}$ Voir d'autres cas comme Jean et son permis de séjour ont expiré (Mel'Cuk, Clas et Polguère, 1995)

${ }^{26}$ Voir aussi les fausses analyses opérées sur Paul est bronzé (cf. *Paul est bronzé. Elle est sèche).

${ }^{27}$ Qui ont le vent en poupe actuellement (cf. Blumenthal et Hausmann, 2006).

${ }^{28} \mathrm{Cf}$. le recours à la traduction.

${ }^{29}$ La précision est importante, mais généralement ignorée. Il est parfaitement possible d'avoir deux synonymes différents, mais qui se laissent coiffer par un synonyme plus général.

${ }^{30}$ Voir « la cartographie de la polysémie verbale » qu'en a tirée François (2007).

${ }^{31} \mathrm{Cf}$. la note précédente sur la synonymie.

${ }^{32}$ C'est avec grand plaisir que nous détournons ludiquement une partie du titre de Constantin de Chanay et RémiGiraud (à paraître) : il s'agit bien de prendre la polysémie au mot !

${ }^{33}$ Wierzbicka (1985) dirait que ce n'est pas un arbre !

${ }^{34}$ Toute stabilisation suppose du temps. Voir actuellement la stabilisation de la lecture métaphorique de tsunami. 
ISBN 978-2-7598-0358-3, Paris, 2008, Institut de Linguistique Française

Conférences plénières

DOI $10.1051 / \mathrm{cmlf0} 834$

${ }^{35}$ L'autre lecture se place bien entendu dans le champ des relations lexicales. 